\title{
PARALLEL KÄHLER SUBMANIFOLDS OF QUATERNIONIC KÄHLER SYMMETRIC SPACES
}

\author{
DMitri V. Alekseevsky, Antonio J. Di Scala \\ AND STEFANO MARCHIAFAVA
}

(Received November 27, 2003, revised August 12, 2004)

\begin{abstract}
The non totally geodesic parallel $2 n$-dimensional Kähler submanifolds of the $n$-dimensional quaternionic projective space were classified by K. Tsukada. Here we give the complete classification of non totally geodesic immersions of parallel $2 m$-dimensional Kähler submanifolds in a quaternionic Kähler symmetric space of non zero scalar curvature, i.e., in a Wolf space or in its non compact dual. They are exhausted by parallel Kähler submanifolds of a totally geodesic submanifold which is either an Hermitian symmetric space or a quaternionic projective space.
\end{abstract}

1. Introduction. Let $\left(\tilde{M}^{4 n}, \tilde{g}, Q\right)$ be a quaternionic Kähler manifold with metric $\tilde{g}$ and parallel quaternionic structure $Q$. A submanifold $M^{2 m}$ together with a section $J_{1} \in$ $\left.\Gamma(Q)\right|_{M}$ such that $J_{1}^{2}=-1$ and $J_{1} T M=T M$ is called Kähler if $J_{1}$ is parallel with respect to the Levi-Civita connection of $\tilde{g}$. We

will study parallel Kähler submanifolds of a quaternionic Kähler symmetric space $\tilde{M}$ of non zero scalar curvature, that is, Kähler submanifolds $M$ with parallel second fundamental form $h$ in a Wolf space or in its non compact dual. In the case when $\operatorname{dim}(\tilde{M})=2 \operatorname{dim} M$, we prove that any curvature invariant and intrinsically locally symmetric Kähler submanifold is parallel, and hence extrinsically symmetric.

Any parallel submanifold $M$ of a Riemannian manifold $\tilde{M}$ is curvature invariant. Furthermore, a curvature invariant, in particular a parallel, maximal Kähler submanifold of a quaternionic Kähler manifold is also normal curvature invariant. Using these properties, we derive the following result from Naitoh's theorem 2.6 in the next section.

THEOREM 1.1. Any curvature invariant (in particular, any parallel) Kähler submanifold $M^{2 n}$ of the maximal dimension $2 n$ of a quaternionic Kähler symmetric space $\tilde{M}^{4 n}$ different from the $n$-dimensional quaternionic projective space $\boldsymbol{H} P^{n}, \tilde{M}^{4 n} \neq \boldsymbol{H} P^{n}$, is totally geodesic.

We recall that a submanifold $M$ of a Riemannian manifold $\tilde{M}$ is called full if $M$ is not contained in a proper totally geodesic submanifold $\bar{M}$ of $\tilde{M}$ and is called 1-full (according

2000 Mathematics Subject Classification. Primary 53C26; Secondary 53C55, 53C40.

Key words and phrases. Quaternionic Kähler manifolds, Kähler submanifolds, totally complex submanifolds.

Supported by a EPSRC Grant GR/R69174, by Italian MURST, under the national project "Geometria delle varietà reali e complesse", and by G.N.S.A.G.A. of C.N.R. (Italy).

The third author is a member of EDGE, Research Training Network HPRN-CT-2000-00101, supported by the European Human Potential Programme. 
to Tsukada [Tsu1]) if the first normal bundle $N^{1} M=h(T M, T M)$ of $M$ coincides with the normal bundle $T^{\perp} M$ of $M$ in $\tilde{M}$.

We associate with a Kähler submanifold $M^{2 m}$ of $\tilde{M}^{4 n}$, of arbitrary dimension $2 m$, a symmetric 3-form $C$, called the shape tensor, and prove the following theorem.

THEOREM 1.2. Let $\left(M^{2 m}, J\right)$ be a geodesically complete parallel Kähler submanifold of a quaternionic Kähler symmetric space $\tilde{M}^{4 n}$ and $\bar{M}$ the minimal totally geodesic submanifold of $\tilde{M}$ containing $M$.

1) If the shape tensor $C$ of $M$ vanishes at one point, then $\bar{M}$ is an Hermitian symmetric space and $M$ is a full parallel Kähler submanifold of $\bar{M}$.

2) If $C \neq 0$, then $\bar{M}=\boldsymbol{H} P^{m}$ and $\left(M^{2 m}, J\right)$ is a Hermitian symmetric manifold with parallel cubic line bundle, that is a product $Q_{m-1} \times C P^{1}$ of the complex quadric $Q_{m-1} \subset$ $C P^{m}$ and the projective line $C P^{1}$, or one of the following exceptional Hermitian symmetric spaces: $\boldsymbol{C} P^{1} \times C P^{1}, C P^{1} \times C P^{1} \times C P^{1}, S p_{2} / U_{2} \times C P^{1}, C P^{1}, S p_{3} / U_{3}, S U_{6} / S\left(U_{3} \times U_{3}\right)$, $\mathrm{SO}_{12} / U_{6}, E_{7} / T^{1} \cdot E_{6}$, with the canonical Tsukada imbedding into $\boldsymbol{H}^{m}$ as described in [Tsu2].

Thus, the classification of parallel Kähler submanifolds of type 1) in a quaternionic Kähler symmetric space reduces to a description of parallel Kähler submanifolds of Hermitian symmetric spaces.

The classification of parallel Kähler submanifolds of $C P^{N}$ was first obtained by Nakagawa and Takagi [NT].

THEOREM 1.3 ([NT]). The only full parallel Kähler submanifolds of a complex projective space are, up to isometries, the images of the Veronese imbedding of the projective space $P V$ associated with $V=C^{n+1}$ into the projectivization $P S^{2} V$ of the symmetric square $S^{2} V$ defined by

$$
\begin{aligned}
\varphi: \quad & \boldsymbol{C} P^{n}=P V \rightarrow P S^{2} V \\
& {[v]=\boldsymbol{C} v \quad \mapsto[v \otimes v], }
\end{aligned}
$$

of the Segre imbedding defined by

$$
\begin{aligned}
\psi: \quad \boldsymbol{C} P^{n} \times \boldsymbol{C} P^{n^{\prime}}=P V \times P V^{\prime} & \rightarrow P\left(V \otimes V^{\prime}\right) \\
\left([v],\left[v^{\prime}\right]\right) & \mapsto\left[v \otimes v^{\prime}\right],
\end{aligned}
$$

or of the first canonical imbedding of compact irreducible Hermitian symmetric spaces of rank 2, i.e., $Q_{n}, G_{2}\left(C^{n+2}\right), S O_{10} / S U_{5}$ and $E_{6} / \operatorname{Spin}_{10} \cdot T$.

The classification of all parallel Kähler submanifolds of a Hermitian symmetric space was established by Tsukada [Tsu1]. He proved that any such submanifold is a product of Veronese submanifolds, Segre submanifolds, canonical Kaehler imbeddings of compact Hermitian symmetric spaces of rank two and trivial factors (defined by the identity map). The Theorem in [Tsu1, p. 130] implies the following 
THEOREM 1.4. There is no full parallel (proper) Kähler submanifold $M$ in a Hermitian symmetric space $\tilde{M}$ having no factor isometric to $C P^{N}$. Any full parallel Kähler submanifold of $\boldsymbol{C} P^{n_{1}} \times C P^{n_{2}}$ has the form $\psi_{1}\left(M_{1}\right) \times \psi_{2}\left(M_{2}\right)$, where $\psi_{i}\left(M_{i}\right) \subset C P^{n_{i}}$ is one of the immersions in Theorem 1.3.

Tsukada [Tsu1] proved that any parallel Kähler submanifold of a Hermitian symmetric space of non compact type is totally geodesic.

These results together give the full classification of non totally geodesic parallel Kähler submanifolds in a quaternionic Kähler symmetric space. A classification of maximal totally geodesic Kähler submanifolds of Wolf spaces in term of Satake diagrams was given by Takeuchi [Tak]. See also Section 6.

The authors like to thank heartily the referee for useful remarks.

\section{Preliminaries.}

2.1. Gauss, Codazzi-Mainardi and Ricci equations. Let $M$ be a submanifold of a Riemannian manifold $\tilde{M}$. We denote by $h: T M \times T M \rightarrow T^{\perp} M$ the second fundamental form of $M$, and by $A^{\xi}$ the shape operator in the direction of a normal vector $\xi \in T^{\perp} M$ such that

$$
\begin{aligned}
\tilde{\nabla}_{X} Y & =\nabla_{X} Y+h(X, Y), \\
\tilde{\nabla}_{X} \xi & =\nabla_{X}^{\perp} \xi-A^{\xi} X,
\end{aligned}
$$

where $X \in T M, Y \in \Gamma T M$ and $\xi \in T^{\perp} M$. Here $\tilde{\nabla}, \nabla, \nabla^{\perp}$ are the Levi-Civita connection of $\tilde{M}$ and the induced connections in $T M$ and $T^{\perp} M$, respectively.

For $X, Y \in T_{X} M$ we decompose the curvature operator $\tilde{R}_{X, Y}$ as

$$
\tilde{R}_{X Y}=R_{X Y}^{T T}+R_{X Y}^{\perp T}+R_{X Y}^{T \perp}+R_{X Y}^{\perp},
$$

according to the decomposition

$$
\operatorname{End}\left(T_{x} \tilde{M}\right)=\operatorname{End}\left(T_{x} M\right)+\operatorname{Hom}\left(T_{x} M, T_{x}^{\perp} M\right)+\operatorname{Hom}\left(T_{x}^{\perp} M, T_{x} M\right)+\operatorname{End}\left(T_{x}^{\perp} M\right) .
$$

Then we have the following Gauss-Codazzi equations:

(Gauss)

$$
R_{X Y}^{\top \top}=R_{X Y}-h_{X} h_{Y}^{t}+h_{Y} h_{X}^{t}=R_{X Y}-\sum_{i} A^{\xi_{i}} X \wedge A^{\xi_{i}} Y
$$

(Codazzi-Mainardi) $\quad R_{X Y}^{\perp \top} Z=\left(\nabla_{X}^{\prime} h\right)(Y, Z)-\left(\nabla_{Y}^{\prime} h\right)(X, Z)$,

(Ricci)

$$
R_{X Y}^{\perp \perp} \xi=R_{X Y}^{\perp} \xi-\sum_{i}\left\langle X,\left[A^{\xi_{i}}, A^{\xi}\right] Y\right\rangle \xi_{i}
$$

where $\xi_{i}$ is an orthonormal basis of $T^{\perp} M, X, Y \in T M, \xi \in T^{\perp} M, R, R^{\perp}$ are the curvature tensors of the connections $\nabla, \nabla^{\perp}$, and $\nabla^{\prime}$ is the connection in $T^{\perp} M \otimes S^{2} T M$ induced by $\nabla^{\perp}$ and $\nabla$, respectively. (We identify a bivector $X \wedge Y$ with the skew-symmetric operator $Z \mapsto\langle Y, Z\rangle X-\langle X, Z\rangle Y$.) 
2.2. Parallel submanifolds of symmetric spaces.

DEFINITION 2.1. A submanifold $M$ of a Riemannian manifold $\tilde{M}$ is called parallel if it has parallel second fundamental form $h$, i.e., $\nabla^{\prime} h=0$.

DEFINITION 2.2. A subspace $V \subset T_{x} \tilde{M}$ of a tangent space of a Riemannian manifold $\tilde{M}$ is called curvature invariant if

$$
\tilde{R}(V, V) V \subset V
$$

A submanifold $M$ of $\tilde{M}$ is called curvature invariant if each tangent space $T_{x} M$ is curvature invariant and it is called normal curvature invariant if each normal space $T_{x}^{\perp} M$ is curvature invariant.

It follows from Codazzi-Mainardi equation that any parallel submanifold $M$ of a Riemannian manifold $\tilde{M}$ is curvature invariant.

DEFINITION 2.3. A submanifold $M$ of a Riemannian manifold $\tilde{M}$ is called 1-full if the first normal bundle $N^{1} M=h(T M, T M)$ coincides with the normal bundle $T^{\perp} M$.

Definition 2.4. Let $\tilde{M}=G / K$ be a homogeneous Riemannian manifold. Fix an orbit $\mathcal{V}$ of the isometry group $G$ in the Grassmann bundle $\operatorname{Gr}_{k}(T \tilde{M})$ of tangent $k$-planes of $\tilde{M}$. If a $k$-plane $V \in \mathcal{V}$ (resp. if the orthogonal plane $V^{\perp}, V \in \mathcal{V}$ ) is curvature invariant, then $\mathcal{V}$ is called curvature invariant (resp. normal curvature invariant).

A $k$-dimensional submanifold $M \subset \tilde{M}$ is called a $\mathcal{V}$-submanifold if $T_{x} M \in \mathcal{V}$ for any $x \in M$. Obviously, if $\mathcal{V}$ is (normal) curvature invariant, then any $\mathcal{V}$-submanifold is (normal) curvature invariant.

DEFINITION 2.5. A submanifold $M$ of a Riemannian manifold $\tilde{M}$ is called extrinsically symmetric if for any point $x \in M$ there exists an involutive isometry (symmetry) $s_{x}$ of $\tilde{M}$ preserving $M$ such that $s_{x}(x)=x$ and its differential at $x$ satisfies

$$
\left.\left(s_{x}\right)_{*}\right|_{T_{X} M}=-\mathrm{Id},\left.\quad\left(s_{x}\right)_{*}\right|_{T_{x}^{\perp} M}=\mathrm{Id} .
$$

We recall the following theorem of Naitoh [Na2].

THEOREM 2.6 (H. Naitoh). Let $\tilde{M}$ be a simply connected Riemannian symmetric space. A submanifold $M$ of $\tilde{M}$ is parallel and normal curvature invariant if and only if it is extrinsically symmetric.

Proof. Let $M \subset \tilde{M}$ be an extrinsically symmetric submanifold. Remark that the symmetry $s_{x}$ acts as - Id on any tensor space $T_{x}^{\otimes^{p}} \otimes T_{x}^{\perp \otimes^{q}}$, where $p$ is odd. On the other hand, it preserves the tensor $\nabla^{\prime} h \in T_{x}^{\otimes^{3}} \otimes T_{x}^{\perp}$ and the curvature tensor $\tilde{R}$ at $x$. This implies that an extrinsically symmetric submanifold is parallel and normal curvature invariant. Conversely, if $M$ is parallel and normal curvature invariant, then the automorphism $\left(s_{x}\right)_{*} \in \operatorname{Gl}\left(T_{x} \tilde{M}\right)$ defined by (1) preserves the curvature tensor $\tilde{R}_{x}$, and hence can be extended to an involutive isometry $s$ of $\tilde{M}$. Now the inverse statement follows from a remarkable theorem of Strübing $[\mathrm{Str}]$. 
THEOREM 2.7 (W. Strubing). Let $M$ be a parallel submanifold of a Riemannian manifold $\tilde{M}$ and $s$ an isometry of $\tilde{M}$ which preserves a point $x \in M$ and satisfies (1). Then $s$ preserves any geodesic $\gamma=\gamma(t)$ of $M$ with $\gamma(0)=x$ : $s(\gamma(t))=\gamma(-t)$. $\tilde{M}:$

The proof follows from the Frenet formulas for the curve $\gamma(t)$ considered as a curve in

$$
\tilde{\nabla}_{\dot{\gamma}}\left(\begin{array}{c}
E_{1} \\
\cdot \\
\cdot \\
\cdot \\
E_{r}
\end{array}\right)=\left(\begin{array}{cccccc}
0 & k_{1} & 0 & \ldots & 0 & 0 \\
-k_{1} & 0 & k_{2} & \ldots & 0 & 0 \\
0 & -k_{2} & 0 & \cdots & \ldots & \cdots \\
\cdots & \cdots & \ldots & \cdots & \cdots & \ldots \\
0 & 0 & 0 & \cdots & 0 & k_{r-1} \\
0 & 0 & 0 & \cdots & -k_{r-1} &
\end{array}\right)\left(\begin{array}{c}
E_{1} \\
\cdot \\
\cdot \\
\cdot \\
E_{r}
\end{array}\right)
$$

where $E_{1}, \ldots, E_{r}$ is an orthonormal Frenet frame along $\gamma$ obtained from the fields $\dot{\gamma}, \ddot{\gamma}, \dddot{\gamma}, \ldots$ by Gram-Schmidt process, $k_{1}, \ldots, k_{r-1}$ are constants ("curvatures") and, moreover, $E_{1}=$ $\dot{\gamma}, E_{3}, E_{5},\left.\ldots \in \Gamma(T M)\right|_{\gamma}$ are $\nabla$-parallel fields and $E_{2}=h(\dot{\gamma}, \dot{\gamma}) /|h(\dot{\gamma}, \dot{\gamma})|, E_{4}, E_{6}, \ldots \in$ $\Gamma\left(T^{\perp} M\right)_{\left.\right|_{\gamma}}$ are $\nabla^{\perp}$-parallel fields along $\gamma$. Indeed, the Frenet frame along $\gamma(-t)$ and $s_{*} E_{i}(t)$ satisfy Frenet equations with the same initial conditions $(-1)^{i} E_{i}(0)$.

Now we state the following fundamental result by Naitoh, which shows that up to a short list of exceptions, a parallel normal curvature invariant (or, equivalently, extrinsically symmetric) $\mathcal{V}$-submanifold of a symmetric space is in fact totally geodesic.

THEOREM 2.8 (H. Naitoh [Na3]). Let $\tilde{M}=G / K$ be a compact simply connected symmetric space with simple isometry group $G$, and $\mathcal{V}$ is an orbit of $G$ in $\operatorname{Gr}_{k}(T \tilde{M})$ which is curvature invariant and normal curvature invariant. Then any $\mathcal{V}$-submanifold is totally geodesic with the exception of the following cases:

(a) $\tilde{M}=S^{n}=S O(n+1) / S O(n), 1 \leq k<n$,

(b) $\tilde{M}=C P^{n}, \mathcal{V}$ is the set of complex $2 k$-subspaces,

(c) $\tilde{M}=C P^{n}, \mathcal{V}$ is the set of totally real $n$-subspaces,

(d) $\tilde{M}=\boldsymbol{H} P^{n}, \mathcal{V}$ is the set of totally complex $2 n$-subspaces,

(e) $\tilde{M}=G / K$ is an irreducible symmetric space and $\mathcal{V}=G T$, where $T$ is the tangent space to an irreducible symmetric $R$-space (i.e., the geometries associated with irreducible symmetric $R$-spaces).

The statement remains true also for non compact dual of $G / K$ [BENT].

The following result will be used in Section 5.

THEOREM 2.9 (H. Naitoh [Na4]). Let $M$ be a parallel submanifold of a symmetric space $\tilde{M}$. If the first osculating space $O_{x}^{1} M=T_{x} M+h\left(T_{x}, T_{x}\right)$ at some point $x \in M$ is curvature invariant, then $M$ is contained in the totally geodesic submanifold $\bar{M}=\exp \left(O_{x}^{1} M\right)$ of $\tilde{M}$ generated by $O_{x}^{1} M$.

Obviously, $M$ is full in $\bar{M}$. 
3. Kähler submanifolds of quaternionic Kähler manifolds. Let $\left(\tilde{M}^{4 n}, Q, \tilde{g}\right)$ be a quaternionic Kähler manifold, that is, a Riemannian manifold $\left(\tilde{M}^{4 n}, \tilde{g}\right)$ with a $\tilde{\nabla}$-parallel quaternionic structure $Q$, i.e., a rank 3 subbundle of $\operatorname{End}(T \tilde{M})$ locally generated by 3 skewsymmetric almost complex structures $J_{1}, J_{2}, J_{3}=J_{1} J_{2}=-J_{2} J_{1}$. For $n=1$, in the definition we assume that $\left(\tilde{M}^{4}, \tilde{g}\right)$ is an anti-self-dual Einstein manifold.

Recall that the curvature tensor $\tilde{R}$ of a quaternionic Kähler manifold has the form

$$
\tilde{R}=\nu R_{H} P^{n}+\tilde{W},
$$

where $\tilde{W}$ is an $\mathfrak{s p}_{n}$-valued 2-form satisfying the Bianchi identities (the quaternionic Weyl tensor $), v=K / 4 n(n+2)$ is the reduced scalar curvature, which is proportional to the scalar curvature $K$, and

$$
R_{H} P^{n}(X, Y)=\frac{1}{4}\left(X \wedge Y+\sum_{\alpha} J_{\alpha} X \wedge J_{\alpha} Y-2 \sum_{\alpha}\left\langle J_{\alpha} X, Y\right\rangle J_{\alpha}\right),
$$

where $\alpha=1,2,3$ and $\langle\rangle=,\tilde{g}($,$) .$

We recall also that the following identities hold:

$$
\left[\tilde{R}(X, Y), J_{\alpha}\right]=-v\left(\left\langle J_{\gamma} X, Y\right\rangle J_{\beta}-\left\langle J_{\beta} X, Y\right\rangle J_{\gamma}\right),
$$

where $(\alpha, \beta, \gamma)$ is a cyclic permutation of $(1,2,3)$. They are equivalent to the following identities

$$
\tilde{R}\left(J_{\alpha} X, J_{\alpha} Y\right) Z=\tilde{R}(X, Y) Z+v\left(\left\langle J_{\beta} X, Y\right\rangle J_{\beta} Z+\left\langle J_{\gamma} X, Y\right\rangle J_{\gamma} Z\right),
$$

which we will need later on.

Definition 3.1. A submanifold $M^{2 m}$ of a quaternionic Kähler manifold $\left(\tilde{M}^{4 n}, Q, \tilde{g}\right)$ together with a section $\left.J_{1} \in \Gamma(Q)\right|_{M}$ such that $J_{1}^{2}=-I d$ and $J_{1}(T M)=T M$ is called

1) a Kähler submanifold if $J_{1}$ is $\tilde{\nabla}$-parallel,

2) a totally complex submanifold if $J_{2}(T M) \perp T M$, where $J_{2} \in Q$ is a complex structure anticommuting with $J_{1}$.

The Kähler submanifold $M^{2 m}$ considered as a manifold with the induced Riemannian metric $g=\tilde{g}_{\mid M}$ and the almost complex structure $J=J_{1 \mid T M}$ is a Kähler manifold.

Recall that if the scalar curvature of $(\tilde{M}, \tilde{g})$ is not zero, then a Kähler submanifold $M^{2 m}, m>1$, is totally complex ([AM2]). In particular, $m \leq n$. A Kähler submanifold of maximal possible dimension $2 n$ is called maximal.

Let $\left(M^{2 m}, J_{1}\right)$ be a Kähler submanifold of a quaternionic Kähler manifold $\tilde{M}^{4 n}$. We fix a local section $\left.J_{2} \in \Gamma(Q)\right|_{M}$ such that $J_{2}^{2}=-1$ and $J_{1} J_{2}=-J_{2} J_{1}$. One can check that

$$
\tilde{\nabla}_{V} J_{2}=\omega(V) J_{3},
$$

where $J_{3}=J_{1} J_{2}$ and $\omega$ is a local 1 -form on $M$. As in [AM1], we associate with the second fundamental form $h$, a (local) (0,3)-tensor field $C$ on $M$, called the shape tensor, defined by

$$
C(X, Y, Z):=\left\langle J_{2} h(X, Y), Z\right\rangle \text {. }
$$


It is symmetric with respect to $X, Y, Z$ and satisfies the following identities:

$$
C(X, Y, J Z)=C(J X, Y, Z)=C(X, J Y, Z),
$$

which means that the associated endomorphism $C_{X}, X \in T M$, defined by

$$
\left\langle C_{X} Y, Z\right\rangle=C(X, Y, Z)
$$

anticommutes with $J$.

If $J_{2}^{\prime}=\cos \theta J_{2}+\sin \theta J_{1} J_{2}$ is another section, then the associated shape tensor $C^{\prime}$ is related to $C$ by

$$
C_{X}^{\prime}=\cos \theta C_{X}+\sin \theta J_{1} \circ C_{X}
$$

This implies that the $\mathfrak{u}_{m}$-valued 2-form $[C, C](X, Y):=\left[C_{X}, C_{Y}\right]$ is globally defined and satisfies the Bianchi identities.

We define the $(0,4)$-tensor field $P$ as follows:

$$
P(V ; X, Y, Z)=\left(\nabla_{V} C\right)(X, Y, Z)+\omega(V) C(X, Y, J Z)
$$

which is symmetric with respect to $X, Y, Z$.

PROPOSITION 3.2. Let $\left(M^{2 m}, J_{1}\right)$ be a curvature invariant Kähler submanifold of a quaternionic Kähler symmetric space. Then

1) the tangential part $R^{T T}$ of the curvature tensor $\tilde{R}$ of $\tilde{M}$ is parallel and the tensor $[C, C]$ satisfies the second Bianchi identity:

$$
\nabla R^{T T}=0, \quad \operatorname{cycl}\left(\nabla_{Z}[C, C]\right)(X, Y)=0
$$

2) If $M$ is parallel, then $P \equiv 0$.

Proof. The proof is the same as for the case $n=m$, which was done in [AM1].

The following Lemma describes the relation between the covariant derivative of $C$ and the tensor $P$.

LEMMA 3.3. Let $\left(M^{2 m}, J_{1}\right)$ be a totally complex submanifold of a quaternionic Kähler manifold $\tilde{M}^{4 n}$. Then the covariant derivative of the shape tensor $C$ is given by

$$
-\left(\nabla_{V} C\right)(X, Y, Z)=\left\langle\left(\nabla_{V}^{\prime} h\right)(X, Y), J_{2} Z\right\rangle+\omega(V) C(X, Y, J Z)+\left\langle h(X, Y), J_{2} h(V, Z)\right\rangle
$$

or, equivalently,

$$
-P(V ; X, Y, Z)=\left\langle\left(\nabla_{V}^{\prime} h\right)(X, Y), J_{2} Z\right\rangle+\left\langle h(X, Y), J_{2} h(V, Z)\right\rangle
$$

for any tangent vectors $X, Y, Z, V$. 
Proof. We extend vectors $X, Y, Z \in T_{X} M$ to local tangent vector fields on $M$ such that $\nabla_{V} X=\nabla_{V} Y=\nabla_{V} Z=0$ at $x \in M$. Then we have

$$
\begin{aligned}
-\left(\nabla_{V} C\right) & (X, Y, Z)=-V C(X, Y, Z)=V\left\langle h(X, Y), J_{2} Z\right\rangle \\
& =\left\langle\nabla_{V}^{\perp} h(X, Y), J_{2} Z\right\rangle+\left\langle h(X, Y), \nabla_{V}^{\perp} J_{2} Z\right\rangle \\
& =\left\langle\left(\nabla_{V}^{\prime} h\right)(X, Y), J_{2} Z\right\rangle+\left\langle h(X, Y), \tilde{\nabla}_{V} J_{2} Z\right\rangle \\
& =\left\langle\left(\nabla_{V}^{\prime} h\right)(X, Y), J_{2} Z\right\rangle+\left\langle h(X, Y),\left(\tilde{\nabla}_{V} J_{2}\right) Z+J_{2} \tilde{\nabla}_{V} Z\right\rangle \\
& =\left\langle\left(\nabla_{V}^{\prime} h\right)(X, Y), J_{2} Z\right\rangle+\left\langle h(X, Y), \omega(V) J_{3} Z+J_{2} h(V, Z)\right\rangle \\
& =\left\langle\left(\nabla_{V}^{\prime} h\right)(X, Y), J_{2} Z\right\rangle+\left\langle h(X, Y),-\omega(V) J_{2} J_{1} Z+J_{2} h(V, Z)\right\rangle \\
& =\left\langle\left(\nabla_{V}^{\prime} h\right)(X, Y), J_{2} Z\right\rangle+\omega(V) C(X, Y, J Z)+\left\langle h(X, Y), J_{2} h(V, Z)\right\rangle .
\end{aligned}
$$

COROLlary 3.4. 1) Assume that at some point $x \in M$ the subspace $\left(\nabla_{T_{x} M}^{\prime} h\right)$ $\left(T_{x} M, T_{x} M\right)$ is orthogonal to $J_{2} T_{x} M$. Then $P_{x}=0$ and the first normal space $N_{x}^{1}=$ $h\left(T_{x} M, T_{x} M\right)$ is totally complex, i.e., $J_{1} N_{x}^{1}=N_{x}^{1}$ and $J_{2} N_{x}^{1}$ is orthogonal to $N_{1 x}$.

2) Assume that $M$ is curvature invariant and the first normal space $N_{x}^{1}$ at some point $x \in M$ is totally complex. Then $P_{x}(V ; X, Y, Z)=\left\langle\left(\nabla_{V}^{\prime} h\right)_{x}(X, Y), J_{2} Z\right\rangle$ is symmetric in all arguments.

Proof. 1) The first term on the right member of (4) vanishes. Hence $P_{x}(V ; X, Y, Z)$ $=-\left\langle h(X, Y), J_{2} h(V, Z)\right\rangle$ is symmetric in all arguments. Since $P_{x}(X, X, X, X)=$ $\left\langle-h(X, X), J_{2} h(X, X)\right\rangle=0$, we get the conclusion.

2) By taking Codazzi-Mainardi equation into account, it is obvious.

THEOREM 3.5. Let $\left(M^{2 m}, J_{1}\right)$ be a totally complex submanifold of a quaternionic Kähler manifold $\tilde{M}^{4 n}$. Assume that $\left\langle\left(\nabla_{V}^{\prime} h\right)(X, Y), J_{2} Z\right\rangle=0$ for any $X, Y, Z, V \in T M$, which is true if $M$ is parallel. Then the first normal bundle $N^{1} M=h(T M, T M)$ is totally complex, i.e., $\left\langle h(X, Y), J_{2} h(V, Z)\right\rangle=0$ and the tensor field $P=0$.

Assume moreover that the reduced scalar curvature $v$ of $\tilde{M}^{4 n}$ is not zero. Then there are two cases:

1) $C=0$ at some point and then $C \equiv 0$, which means that $N^{1} M \perp J_{2} T M$, or

2) $C \neq 0$ and then $M$ is a locally symmetric Hermitian manifold with parallel cubic line bundle of type $v$ ([AM1]). More precisely, $M$ is locally isometric to one of the symmetric spaces: $S=Q_{n-1} \times \boldsymbol{C} P^{1}, \boldsymbol{C} P^{1} \times \boldsymbol{C P} P^{1}, \boldsymbol{C} P^{1} \times \boldsymbol{C} P^{1} \times \boldsymbol{C P} P^{1}, S p_{2} / U_{2} \times C P^{1}, C P^{1}, S p_{3} / U_{3}$, $S U_{6} / S\left(U_{3} \times U_{3}\right), S O_{12} / U_{6}, E_{7} / T^{1} \cdot E_{6}$ or its non compact dual.

Proof. By Corollary 3.4, the tensor $P$ vanishes, that is,

$$
P(V ; X, Y, Z)=\left(\nabla_{V} C\right)(X, Y, Z)+\omega(V) C(X, Y, J Z) \equiv 0 .
$$

It was shown in [AM1] that if $C \neq 0$ at least at one point, then this condition means that the tensor field $C$ generates a parallel holomorphic line bundle in the space of cubic symmetric forms of type $(3,0)$ such that the induced connection has curvature $R^{L}=i \nu g \circ J$ (parallel 
cubic line bundle of type v). All such Kähler manifolds are locally symmetric and locally isometric to one of the symmetric spaces described in [AM1, Thm. 3.14].

4. Characterization of maximal parallel Kähler submanifolds of a quaternionic Kähler symmetric space. In this section we give a characterization of maximal parallel Kähler submanifolds $M^{2 n}$ of a quaternionic Kähler symmetric space $\tilde{M}^{4 n}$, of non zero scalar curvature.

THEOREM 4.1. Let $M^{2 n} \subset \tilde{M}^{4 n}$ be a complete maximal Kähler submanifold of a quaternionic Kähler symmetric space $\tilde{M}^{4 n}$ of non zero scalar curvature. Then the following properties are equivalent:

(i) $M$ is curvature invariant and locally symmetric.

(ii) $M$ is parallel.

(iii) $M$ is extrinsically symmetric.

PROOF. For proof we need the following lemma.

LEMMA 4.2 ([AM1, Prop. 2.8]). Any curvature invariant maximal Kähler submanifold $\left(M^{2 n}, J\right)$ of a quaternionic Kähler manifold $\tilde{M}^{4 n}$ is normal curvature invariant.

PROOF. The proof follows from the following identity which implies that the curvature tensor $\tilde{R}$ is invariant under the automorphism $J_{2}$ :

$$
\left\langle\tilde{R}\left(J_{2} X, J_{2} Y\right) J_{2} Z, J_{2} W\right\rangle=\langle\tilde{R}(X, Y) Z, W\rangle
$$

for all $X, Y, Z, W \in T \tilde{M}$.

Proof of THE TheOrEm 4.1. The equivalence (ii) $\Leftrightarrow$ (iii) follows from the Lemma and Theorem 2.6. (ii) $\Rightarrow$ (i) is well-known.

Thus, it remains to prove that (i) $\Rightarrow$ (ii). Assume that $M$ is curvature invariant and locally symmetric. Then, by Proposition 2.13 in [AM1, page 887] the tensor field [C, $C$ ] is parallel, i.e., $\nabla[C, C]=0$. We associate to the shape operator $A$ the tensor $[A, A] \in$ $\Gamma\left(\Lambda^{2} T^{\perp} M \otimes \Lambda^{2} T M\right)$ by $[A, A](\xi, \eta)=\left[A^{\xi}, A^{\eta}\right]$ for $\xi, \eta \in T^{\perp} M$.

We need the following lemma.

LEMMA 4.3. Let $M^{2 n}$ be a maximal Kähler submanifold of a quaternionic symmetric space $\tilde{M}^{4 n}, v \neq 0$, and $A^{\xi}$ its shape operator. Then the following holds:

$$
\left(\nabla_{Z}[C, C]\right)\left(J_{2} \xi, J_{2} \eta\right) W=\left(\nabla_{Z}^{\prime}[A, A]\right)(\xi, \eta) W .
$$

Proof of Lemma. For $\xi, \eta \in J_{2} T_{x} M$ and $Z, W \in T_{x} M$, we have

$$
\left(\nabla_{Z}[C, C]\right)\left(J_{2} \xi, J_{2} \eta\right) W=\nabla_{Z}(C \circ C)\left(J_{2} \xi, J_{2} \eta\right) W-\nabla_{Z}(C \circ C)\left(J_{2} \eta, J_{2} \xi\right) W .
$$

We have

$$
\nabla_{Z}(C \circ C)\left(J_{2} \xi, J_{2} \eta\right) W=\left(\left(\nabla_{Z} C\right) \circ C\right)\left(J_{2} \xi, J_{2} \eta\right) W+\left(C \circ\left(\nabla_{Z} C\right)\right)\left(J_{2} \xi, J_{2} \eta\right) W .
$$

By definition it follows that

$$
\left(\nabla_{Z} C\right)_{V} W=\nabla_{Z} C_{V} W-C_{\nabla_{Z} V} W-C_{V} \nabla_{Z} W .
$$


Hence we obtain

$$
\begin{aligned}
& \nabla_{Z}(C \circ C)\left(J_{2} \xi, J_{2} \eta\right) W=\left(\left(\nabla_{Z}^{\prime} A\right)^{\xi} \circ A^{\eta}\right) W+\left(A^{\xi} \circ\left(\nabla_{Z}^{\prime} A\right)^{\eta}\right) W \\
& -\left(C_{\left(\nabla_{Z} J_{2}\right) \xi} \circ C_{J_{2} \eta}\right) W-\left(C_{J_{2} \xi} \circ C_{\left(\nabla_{Z} J_{2}\right) \eta}\right) W \text {. }
\end{aligned}
$$

Since $\left(\nabla_{Z} J_{2}\right)=\omega(Z) J_{3}$, we get

$$
\left(C_{\left(\nabla_{Z} J_{2}\right) \xi} \circ C_{J_{2} \eta}\right) W+\left(C_{J_{2} \xi} \circ C_{\left(\nabla_{Z} J_{2}\right) \eta}\right) W=0 .
$$

Then,

$$
\begin{aligned}
\nabla_{Z}(C \circ C)\left(J_{2} \xi, J_{2} \eta, W\right) & =\left(\left(\nabla_{Z}^{\prime} A\right)^{\xi} \circ A^{\eta}\right) W+\left(A^{\eta} \circ\left(\nabla_{Z}^{\prime} A\right)^{\eta}\right) W \\
& =\nabla_{Z}^{\prime}(A \circ A)(\xi, \eta, W) .
\end{aligned}
$$

Now, the lemma follows from the above identity.

By using this lemma, we see that (i) implies $\left(\nabla_{Z}^{\prime}[A, A]\right)(\xi, \eta) W=0$. Since $J_{1}$ is parallel, we obtain that $\left(\nabla_{Z}^{\prime}[A, A]\right)\left(\xi, J_{1} \eta\right) W=0$. From these two identities we get

$$
\nabla_{Z}^{\prime}(A \circ A)(\xi, \eta) W=\left(\left(\nabla_{Z}^{\prime} A\right)^{\xi} \circ A^{\eta}\right) W+\left(A^{\xi} \circ\left(\nabla_{Z}^{\prime} A\right)^{\eta}\right) W=0 .
$$

Also, we have

$$
\nabla_{J_{1} Z}^{\prime}(A \circ A)(\xi, \eta) W=\left(\left(\nabla_{J_{1} Z}^{\prime} A\right)^{\xi} \circ A^{\eta}\right) W+\left(A^{\xi} \circ\left(\nabla_{J_{1} Z}^{\prime} A\right)^{\eta}\right) W=0 .
$$

Since $M$ is curvature invariant, it follows that $\left(\nabla_{J_{1} Z}^{\prime} A\right)^{\xi} X=-J_{1}\left(\nabla_{Z}^{\prime} A\right)^{\xi} X$. By using this fact together with the last two identities, we obtain

$$
\left(\left(\nabla_{Z}^{\prime} A\right)^{\xi} \circ A^{\eta}\right) W=\left(A^{\xi} \circ\left(\nabla_{Z}^{\prime} A\right)^{\eta}\right) W=0 .
$$

Now, the theorem is a consequence of the following lemma.

LEMMA 4.4. Let $M$ be a submanifold of a Riemannian manifold and $A$ its shape operator. If

$$
\left(\left(\nabla_{X} A\right)^{\xi} \circ A^{\eta}\right) W=\left(A^{\xi} \circ\left(\nabla_{X} A\right)^{\eta}\right) W=0,
$$

then $M$ is parallel, i.e., $\nabla^{\prime} A=0$.

Proof. We decompose $T M=\mathcal{N} \oplus \mathcal{N}^{\perp}$, where

$$
\mathcal{N}=\bigcap_{\xi \in T M^{\perp}} \operatorname{ker}\left(A^{\xi}\right), \quad \mathcal{N}^{\perp}=\operatorname{span}\left(\bigcup_{\xi \in T M^{\perp}} \operatorname{Image}\left(A^{\xi}\right)\right) .
$$

So, if $Z \in \mathcal{N}^{\perp}$, it follows that $\left(\nabla_{X}^{\prime} A\right)(\xi, Z)=0$. Let $Z \in \mathcal{N}$ be any section. Observe that $\left(\nabla_{X}^{\prime} A\right)^{\xi} Z \in \mathcal{N}$. On the other hand, we have $\left(\nabla_{X}^{\prime} A\right)^{\xi} Z=-A^{\xi} \nabla_{X} Z$. Thus, $\left(\nabla_{X}^{\prime} A\right)^{\xi} Z \in \mathcal{N}^{\perp}$ and then $\left(\nabla_{X}^{\prime} A\right)^{\xi} Z=0$, that is, $A$ is parallel.

\section{Parallel Kähler submanifolds of a symmetric quaternionic Kähler manifold.}

5.1. Reduction to the case of 1-full parallel Kähler submanifolds. Note that the intersection of totally geodesic submanifolds of a Riemannian manifold $\tilde{M}$ is a totally geodesic 
submanifold. Hence we may consider the minimal totally geodesic submanifold $\bar{M}$ containing a given submanifold $M$.

In this subsection we prove the following theorem which reduces the classification of parallel Kähler submanifolds of a quaternionic Kähler symmetric manifold to the classification of 1-full parallel Kähler submanifolds in Hermitian or quaternionic Kähler symmetric spaces.

THEOREM 5.1. Let $\left(M^{2 m}, J\right)$ be a parallel Kähler submanifold of a symmetric quaternionic Kähler manifold $\tilde{M}^{4 n}$ of non zero scalar curvature and $\bar{M}$ the minimal totally geodesic submanifold of $\tilde{M}^{4 n}$ containing $M^{2 m}$.

1) If the shape tensor $C$ of $\left(M^{2 m}, J\right)$ vanishes, then $\bar{M}$ is a totally geodesic Hermitian symmetric space and $\left(M^{2 m}, J\right)$ is a full parallel Kähler submanifold of $\bar{M}$.

2) If $C \neq 0$, and hence $\left(M^{2 m}, J\right)$ is a Kähler manifold with parallel cubic line bundle, then $\bar{M}$ is a quaternionic Kähler symmetric space of dimension $4 m$ and $\left(M^{2 m}, J\right)$ is a full parallel Kähler submanifold of $\bar{M}$.

PROOF. We need the following Lemma.

DEFINITION 5.2. A parallel Kähler submanifold of a symmetric quaternionic Kähler manifold $\tilde{M}^{4 n}$ is called of type 1 ) if the shape tensor $C=0$ and of type 2) otherwise.

LEMMA 5.3. Let $M$ be a parallel Kähler submanifold of a symmetric quaternionic Kähler manifold with non zero scalar curvature.

1) If it is of type 1), then

$$
J_{2} T_{x} M \perp N_{x}^{1} \text { for all } x \in M .
$$

2) If it is of type 2), then

$$
J_{2} T_{x} M=N_{x}^{1} \text { for all } x \in M .
$$

Proof of Lemma 5.3. 1) is obvious, by definition of $C$. Before considering the case 2 ), let state some facts which hold true for any parallel submanifold $M$. As before, we use Latin letters $X, Y, Z, \ldots$ for vector fields in $T M$ and Greek letters $\xi, \eta, \ldots$ for vector fields in $T^{\perp} M$. By hypothesis $\nabla^{\prime} h=0$ we have the identity

$$
\nabla_{X}^{\perp}(h(Y, Z))=h\left(\nabla_{X} Y, Z\right)+h\left(Y, \nabla_{X} Z\right),
$$

and

$$
\tilde{R}(T M, T M) T M \subset T M .
$$

Moreover, by (2) of Lemma 13 of [Na1],

$$
\tilde{R}(T M, T M) N^{1} \subset N^{1} .
$$

(Naitoh proved (7) as follows: the Ricci equation of the parallel submanifold can be written as

$$
\tilde{R}(X, Y) \xi=R^{\perp}(X, Y) \xi-h\left(X, A^{\xi} Y\right)+h\left(A^{\xi} X, Y\right)
$$


and for $\xi=h(Z, T)$, by (5), it follows that

$$
R^{\perp}(X, Y) h(Z, T)=h(R(X, Y) Z, T)+h(Z, R(X, Y) T) .
$$

The conclusion follows immediately).

The proof of the Lemma follows directly from the next two Sublemmas.

SublemMA 5.4. For any parallel Kähler submanifold $M$ one has

$$
\tilde{R}\left(T M, N^{1}\right) T M \subset N^{1} .
$$

Moreover, if $M$ is of type 2), then

$$
J_{2} T M \subset N^{1} .
$$

Proof of Sublemma 5.4. Since $\tilde{M}^{4 n}$ is a symmetric space and the submanifold $M$ is curvature invariant, we have $\left(\tilde{\nabla}_{X} \tilde{R}\right)(Z, U) Y=0$, which can be written as

$$
\begin{aligned}
& \nabla_{X}(\tilde{R}(Z, U) Y)+h(\tilde{R}(Z, U) Y, X) \\
& \quad=\tilde{R}\left(\nabla_{X} Z, U\right) Y+\tilde{R}\left(Z, \nabla_{X} U\right) Y+\tilde{R}(Z, U) \nabla_{X} Y \\
& \quad+\tilde{R}(h(X, Z), U) Y+\tilde{R}(Z, h(X, U)) Y+\tilde{R}(Z, U) h(X, Y) .
\end{aligned}
$$

The projection onto $T^{\perp} M$ of this identity gives

$$
\tilde{R}(h(X, Z), U) Y+\tilde{R}(Z, h(X, U)) Y=h(\tilde{R}(Z, U) Y, X)-\tilde{R}(Z, U) h(X, Y) .
$$

By comparing (10) with the identity obtained by changing $X \rightarrow J_{1} X$ and $U \rightarrow J_{1} U$, and taking account of (2), we deduce the following identity:

$$
\begin{aligned}
\tilde{R}(h(X, Z), U) Y= & (1 / 2)\left[-v\left(\left\langle J_{2} h(X, Z), U\right\rangle J_{2} Y+\left\langle J_{3} h(X, Z), U\right\rangle J_{3} Y\right)\right. \\
& +h(\tilde{R}(Z, U) Y, X)+h(\tilde{R}(Z, J U) Y, J X) \\
& -\tilde{R}(Z, U) h(X, Y)-\tilde{R}(Z, J U) h(J X, Y)] .
\end{aligned}
$$

If $M$ is of type 1), then (8) follows from (11), (6), (7). Let now assume that $M$ is of type 2). We use (11) to compute the first two terms of the Bianchi identity $0=\tilde{R}(h(X, Z), U) Y+$ $\tilde{R}(Y, h(X, Z)) U+\tilde{R}(U, Y) h(X, Z)$. Taking account of (7), we get

$$
\begin{aligned}
& -\left\langle J_{2} h(X, Z), U\right\rangle J_{2} Y-\left\langle J_{3} h(X, Z), U\right\rangle J_{3} Y \\
& \quad+\left\langle J_{2} h(X, Z), Y\right\rangle J_{2} U+\left\langle J_{3} h(X, Z), Y\right\rangle J_{3} U \in N^{1} .
\end{aligned}
$$

Let us assume that at a point $x \in M$ there exists a vector $Y$ such that $J_{2} Y \notin\left(N^{1}\right)^{\perp}$. If $U=J_{1} Y$, then (12) gives

$$
\left\langle J_{3} h(X, Z), Y\right\rangle J_{2} Y-\left\langle J_{2} h(X, Z), Y\right\rangle J_{3} Y \in N^{1}
$$

and, by changing $X \rightarrow J_{1} X$, we get

$$
\left\langle J_{2} h(X, Z), Y\right\rangle J_{2} Y+\left\langle J_{3} h(X, Z), Y\right\rangle J_{3} Y \in N^{1} .
$$

By assumption, there exist vectors $X, Z \in T_{x} M$ such that

$$
\left\langle J_{2} h(X, Z), Y\right\rangle^{2}+\left\langle J_{3} h(X, Z), Y\right\rangle^{2} \neq 0 .
$$


Then (13) and (14) imply that $J_{2} Y, J_{3} Y \in N^{1}$. Now, for any $U \in T M$, (12) gives

$$
\left\langle J_{2} h(X, Z), Y\right\rangle J_{2} U+\left\langle J_{3} h(X, Z), Y\right\rangle J_{3} U \in N^{1},
$$

from which, by comparing with the identity where $U$ is replaced with $J U$, it is easy to deduce that $J_{2} U \in N^{1}$ for any $U \in T M$. (8) follows from (11), (6), (9) and (7).

SublemMA 5.5. If $M$ is of type 2) then

$$
J_{2} N^{1} \subset T M .
$$

Proof of Sublemma 5.5. Let us assume that the vector field $\xi \in N^{1}$. Since $\tilde{R}(Y, \xi) Z \in N^{1}$ by $(8)$, the identity $\left(\tilde{\nabla}_{X} \tilde{R}\right)(Y, \xi) Z=0$ can be rewritten as

$$
\begin{aligned}
\nabla_{X}^{\perp} \tilde{R}(Y, \xi) Z-A^{\tilde{R}(Y, \xi) Z} X= & \tilde{R}\left(\nabla_{X} Y, \xi\right) Z+\tilde{R}\left(Y, \nabla_{X}^{\perp} \xi\right) Z+\tilde{R}(Y, \xi) \nabla_{X} Z \\
& +\tilde{R}(h(X, Y), \xi) Z-\tilde{R}\left(Y, A_{X}^{\xi}\right) Z+\tilde{R}(Y, \xi) h(X, Z) .
\end{aligned}
$$

By using repeatedly (5), (7) and (8), we get

$$
\tilde{R}(h(X, Y), \xi) Z+\tilde{R}(Y, \xi) h(X, Z) \in O^{1}
$$

and, by changing $Y \rightarrow J Y$ and $\xi \rightarrow J \xi$,

$$
\tilde{R}\left(J_{1} h(X, Y), J_{1} \xi\right) Z+\tilde{R}\left(J_{1} Y, J_{1} \xi\right) h(X, Z) \in O_{x}^{1}=T_{x} M+h\left(T_{x} M, T_{x} M\right) .
$$

The last two identities together with (2) imply that

$$
\begin{aligned}
& v\left(\left\langle J_{2} h(X, Y), \xi\right\rangle J_{2} Z+\left\langle J_{3} h(X, Y), \xi\right\rangle J_{3} Z\right. \\
& \left.\quad+\left\langle J_{2} Y, \xi\right\rangle J_{2} h(X, Z)+\left\langle J_{3} Y, \xi\right\rangle J_{3} h(X, Z)\right) \in O_{x}^{1} .
\end{aligned}
$$

Since $J_{2} Z, J_{3} Z \in N^{1}$ by Lemma 5.4 , we conclude that

$$
\left\langle J_{2} Y, \xi\right\rangle J_{2} h(X, Z)+\left\langle J_{3} Y, \xi\right\rangle J_{3} h(X, Z) \in O_{x}^{1} .
$$

Let us assume that there exists a vector $Y \in T_{x} M$ such that $\left\langle J_{2} Y, \xi\right\rangle^{2}+\left\langle J_{3} Y, \xi\right\rangle^{2} \neq 0$. We deduce easily, by comparing (17) with the identity obtained by the change $Y \rightarrow J_{1} Y$, that

$$
J_{2} h(X, Z), J_{3} h(X, Z) \in O^{1} \text { for any } X, Z \in T_{x} M .
$$

On the other hand, by Corollary 3.4, $J_{2} h(X, Z)$ is orthogonal to $N^{1}$. Hence

$$
J_{2} h(X, Z), J_{3} h(X, Z) \in T M \text { for any } X, Z \in T_{x} M,
$$

and (5.5) follows. This finish the proof of Sublemma 5.5 and hence Lemma 5.3.

Now we prove the following Proposition which, together with Lemma 5.3, implies Theorem 5.1.

Proposition 5.6. Let $\left(M^{2 m}, J\right)$ be a parallel Kähler submanifold of a locally symmetric quaternionic Kähler manifold. Then the first osculating space $O_{x}^{1}=T_{x} M+N_{x}^{1}$ at any point $x \in M$ is curvature invariant, i.e.,

$$
\tilde{R}\left(O^{1}, O^{1}\right) O^{1} \subset O^{1} .
$$


REMARK. The proposition remains true if $\tilde{M}$ is a locally symmetric Kähler manifold, whose proof is the same as in the quaternionic Kähler case.

ProOF. The identity $(\tilde{\nabla} \tilde{R})(Y, Z) \xi=0$ can be rewritten as

$$
\begin{aligned}
\nabla_{X}^{\perp}(\tilde{R}(Y, Z) \xi)-A^{\tilde{R}(Y, Z) \xi} X= & \tilde{R}\left(\nabla_{X} Y, Z\right) \xi+\tilde{R}\left(Y, \nabla_{X} Z\right) \xi+\tilde{R}(Y, Z) \nabla_{X}^{\perp} \xi \\
& +\tilde{R}(h(X, Y), Z) \xi+\tilde{R}(Y, h(X, Z)) \xi-\tilde{R}(Y, Z) A^{\xi} X
\end{aligned}
$$

For $\xi \in N^{1}$, by taking account of (6), (7) and (5), this gives

$$
\tilde{R}(h(X, Y), Z) \xi+\tilde{R}(Y, h(X, Z)) \xi \in O^{1} .
$$

By changing $X \rightarrow J_{1} X$ and $Z \rightarrow J_{1} Z$, we have

$$
\tilde{R}\left(J_{1} h(X, Y), J_{1} Z\right) \xi-\tilde{R}(Y, h(X, Z)) \xi \in O^{1} .
$$

By (2), we also have

$$
\begin{aligned}
\tilde{R}\left(J_{1} h(X, Y), J_{1} Z\right) \xi= & \tilde{R}(h(X, Y), Z) \xi+v\left(\left\langle J_{2} h(X, Y), Z\right\rangle J_{2} \xi\right. \\
& \left.+\left\langle J_{3} h(X, Y), Z\right\rangle J_{3} \xi\right) \in T M,
\end{aligned}
$$

which implies

$$
\tilde{R}\left(N^{1}, T M\right) N^{1} \subset O^{1} .
$$

Now the Bianchi identity gives

$$
\tilde{R}\left(N^{1}, N^{1}\right) T M \subset O^{1} .
$$

We rewrite the identity $(\tilde{\nabla} \tilde{R})(Y, \eta) \xi=0$ for $\eta, \xi \in N^{1}$ as follows:

$$
\begin{aligned}
\tilde{\nabla}_{X}(\tilde{R}(Y, \eta) \xi)= & \tilde{R}\left(\nabla_{X} Y, \eta\right) \xi+\tilde{R}\left(Y, \nabla_{X}^{\perp} \eta\right) \xi+\tilde{R}(Y, \eta) \nabla_{X}^{\perp} \xi \\
& +\tilde{R}(h(X, Y), \eta) \xi-\tilde{R}\left(Y, A^{\eta} X\right) \xi-\tilde{R}(Y, \eta) A^{\xi} X .
\end{aligned}
$$

Since the bundle $O^{1}$ is invariant under parallel transport, it follows that $\tilde{R}(h(X, Y), \eta) \xi \in O_{x}^{1}$, and hence

$$
\tilde{R}\left(N_{x}^{1}, N_{x}^{1}\right) N_{x}^{1} \subset O_{x}^{1} .
$$

Formulas (6), (7), (8), (18), (19) and (20) then imply Proposition 5.6.

We also obtain the following corollary, which was proved by Tsukada [Tsu2] in the case of quaternionic projective space.

COROLLARY 5.7. A non totally geodesic parallel totally complex submanifold $\left(M^{2 m}, J_{1}\right)$ of a symmetric quaternionic Kähler manifold $\tilde{M}^{4 n}$ is 1-full if and only if it has maximal dimension, i.e., $n=m$.

PROOF. We have the following orthogonal decomposition:

$$
T \tilde{M}=T M+J_{2}(T M)+N(M),
$$


where $N(M)$ is a quaternionic subbundle. If we assume that $M$ is 1-full, then it follows that $T^{\perp} M=J_{2} T M+N M=N^{1} M$. By 1) of Corollary 3.4, $N^{1} M$ is totally complex, and hence $N M=0$. Vice versa, if $M$ has maximal dimension $n=m$, then $J_{2} T M=T^{\perp} M$. Since $M$ is not totally geodesic, $M$ has type 2) and by Lemma 5.3, we get $N^{1} M=J_{2} T M=T^{\perp} M$.

REMARK 5.8. As a consequence of Proposition 5.6 and Naitoh's Theorem 2.9, it follows that the concept of being 1-full and that of being full are equivalent for a parallel Kähler submanifold of a locally symmetric quaternionic Kähler manifold.

Now we can prove Theorem 5.1. By Proposition 5.6 and Theorem 2.9, the Kähler submanifold $M^{2 m}$ is 1 -full in the totally geodesic submanifold $\bar{M}=\exp \left(O_{x}^{1} M\right)$. In the case 1), $\bar{M}$ is a totally complex totally geodesic submanifold, and hence a Hermitan symmetric space. In the case 2), $\bar{M}$ is a quaternionic Kähler submanifold.

6. Totally geodesic maximal Kähler submanifolds of Wolf spaces. All totally geodesic maximal Kähler submanifolds $M^{2 n}$ of a Wolf space $W=G / K=\tilde{M}^{4 n}$ were classified by Takeuchi in terms of Satake diagrams [Tak]. Here we sketch another approach based on a simple observation that there exists a natural one to one correspondence between such submanifolds and involutive automorphisms of the complex Lie algebra $\mathfrak{g}=\operatorname{Lie}(G)^{C}$, which preserve the canonical ideal $\mathfrak{s p}_{1}$ of the stability Lie algebra $\mathfrak{k}$ and act non trivially on it. Similar ideas can be found in [Wo].

6.1. Lie algebra description of Wolf spaces. Recall that any simple complex Lie algebra $\mathfrak{g}$ determine the Wolf space as follows. Let

$$
\mathfrak{g}=\mathfrak{h}+\sum_{\alpha \in R} \boldsymbol{C} E_{\alpha}
$$

be the Cartan decomposition of the Lie algebra $\mathfrak{g}$ with respect to a Cartan subalgebra $\mathfrak{h}$ and $\Pi=\left\{\alpha_{1}, \ldots, \alpha_{\ell}\right\}$ a system of simple roots of the root system $R$.

We denote by $\mu$ the maximal root of $R$ and by $H_{\mu}=2 /(\mu, \mu) B_{\mu}^{-1}=\left[E_{\mu}, E_{-\mu}\right]$ the corresponding element of $\mathfrak{h}$ such that $\left\{H_{\mu}, E_{ \pm \mu}\right\}$ is the standard basis of the 3-dimensional subalgebra $\mathfrak{a}_{1}=\mathfrak{s p}_{1}^{\mu}(\boldsymbol{C})$. Then $\operatorname{ad}_{H_{\mu}}$ has the eigenvalues $\pm 2, \pm 1,0$ and the corresponding eigenspace decomposition

$$
\mathfrak{g}=\mathfrak{g}_{-2}+\mathfrak{g}_{-1}+\mathfrak{g}_{0}+\mathfrak{g}_{1}+\mathfrak{g}_{2}
$$

gives rise to a gradation of the Lie algebra $\mathfrak{g}$. Moreover, we have

$$
\mathfrak{g}_{ \pm 2}=\boldsymbol{C} E_{ \pm \mu}, \quad \mathfrak{g}_{ \pm 1}=\sum_{\alpha \in \pm R_{1}} \mathfrak{g}_{\alpha}, \quad \mathfrak{g}_{0}=\mathfrak{h}+\sum_{\alpha \in R_{0}} \boldsymbol{C} E_{\alpha}=\mathfrak{g}_{0}^{\prime} \oplus \boldsymbol{C} H_{\mu}
$$

where

$$
R_{1}=\left\{\alpha \in R ; \alpha\left(H_{\mu}\right)=\frac{2(\alpha, \mu)}{(\mu, \mu)}=1\right\}, \quad R_{0}=\{\alpha \in R ;(\alpha, \mu)=0\} .
$$


We put $\varphi_{0}=\exp i \pi\left(\operatorname{ad}_{H_{\mu}}\right)$, which is an involutive automorphism of $\mathfrak{g}$ with eigenspace decomposition

$$
\mathfrak{g}=\mathfrak{g}_{\mathrm{ev}}+\mathfrak{g}_{\text {odd }}=\left(\mathfrak{g}_{-2}+\mathfrak{g}_{0}+\mathfrak{g}_{2}\right)+\left(\mathfrak{g}_{-1}+\mathfrak{g}_{1}\right) .
$$

Since $\varphi_{0}$ commutes with the standard antilinear involution $\tau$ of $\mathfrak{g}$ associated with the Cartan decomposition, which determines the compact real form $\mathfrak{g}^{\tau}=\{X \in \mathfrak{g} ; \tau(X)=X\}, \varphi_{0}$ defines a symmetric decomposition

$$
\mathfrak{g}^{\tau}=\mathfrak{g}_{\mathrm{ev}}^{\tau}+\mathfrak{g}_{\text {odd }}^{\tau}=\left(\mathfrak{s p}_{1}^{\mu}+\mathfrak{g}_{0}^{\prime}\right)^{\tau}+\left(\mathfrak{g}_{-1}+\mathfrak{g}_{1}\right)^{\tau}=\mathfrak{k}+\mathfrak{m}
$$

of the compact Lie algebra $\mathfrak{g}^{\tau}$. We denote by $G$ the adjoint (compact) Lie group with the Lie algebra $\mathfrak{g}^{\tau}$ and by $K=N_{G}\left(\mathfrak{a}_{1}\right)=S p_{1}^{\mu} \cdot K^{\prime}$ the normalizer of the 3-dimensional subalgebra (which is the connected Lie group generated by the subalgebra $\mathfrak{k}=\mathfrak{g}_{\mathrm{ev}}^{\tau}$ ). Then $W=G / K$ is a simply connected irreducible symmetric space $W=G / K$ associated with this symmetric decomposition. Moreover, it has a natural structure of quaternionic Kähler symmetric space, which is called the Wolf space associated with the Lie algebra $\mathfrak{g}$. The quaternionic structure $Q$ in the tangent space $T_{o} W=\mathfrak{g}_{\text {odd }}^{\tau}$ is given by $Q=\left.\operatorname{ad}_{\mathfrak{s p}_{1}^{\mu}}\right|_{\mathfrak{g}_{\text {odd }}^{\tau}}$.

Remark that the pair $(G, K)$ is determined by the grading element $d=H_{\mu}$ of the gradation (21) and the antilinear involution $\tau$ with $\tau d=-d$. Conversely, a pair $(d, \tau)$, where $d$ is the grading element of a gradation (21) with $\operatorname{dim} \mathfrak{g}_{ \pm 2}=1$ and $\tau$ is an antilinear involution of $\mathfrak{g}$ with $\tau d=-d$, defining a compact real form $\mathfrak{g}^{\tau}$ of $\mathfrak{g}$, defines a Wolf space $W=G / K$, and any such pairs are conjugated by an inner automorphism of $\mathfrak{g}$.

6.2. Totally geodesic extrinsically symmetric Kähler submanifolds of a Wolf space. Let $W=G / K$ be a Wolf space associated with a complex simple Lie algebra $\mathfrak{g}$ and $(d=$ $\left.H_{\mu}, \tau\right)$ be the pair that determines $(G, K)$ as above. Since the isotropy group $K=S p_{1}^{\mu} \cdot K^{\prime}$ acts transitively on the unit sphere of all complex structures $J \in Q=\mathrm{ad}_{\mathfrak{a}_{1}} \mid \mathfrak{m}$, any totally geodesic Kähler submanifold $M$ of $W$ containing $o=e K \in W$ is $K$-equivalent to a submanifold $M^{\prime} \ni o$, whose tangent space $T_{o} M$ is invariant under some fixed complex structure $J_{1} \in Q$. We choose as $J_{1}$ the complex structure $J_{1}=\left.\operatorname{ad}_{i H_{\mu}}\right|_{\mathfrak{g}_{\text {odd }}^{\tau}}$. We will call a totally geodesic Kähler submanifold $M$ of $W$ admissible if it contains $o$ and the tangent space $T_{o} M$ is $J_{1}$-invariant.

THEOREM 6.1. Let $W=G / K$ be a Wolf space associated with a complex simple Lie algebra $\mathfrak{g}, d=H_{\mu}$ be the grading element of the gradation (21) and $\tau$ be the antilinear involution defining the compact real form Lie $G=\mathfrak{g}^{\tau}$ of $\mathfrak{g}$.

1) There is a natural one-to-one correspondence between

i) involutive automorphisms $\sigma$ of $\mathfrak{g}$ which commute with $\tau$ and satisfy condition $\sigma\left(E_{ \pm \mu}\right)=-E_{ \pm \mu}$, and

ii) (connected) admissible totally geodesic extrinsically symmetric Kähler submanifolds $M(\sigma)$ of $W=G / K$ given by $M(\sigma)=W^{s_{\sigma}}$, where $W^{s_{\sigma}} \ni o$ is the connected component of the fixed points set of the symmetry $s_{\sigma}: W \ni$ $a K \mapsto \sigma(a) K$. Moreover, $\operatorname{dim} M(\sigma)=(1 / 2) \operatorname{dim} W$.

2) Submanifolds $M(\sigma)$ and $M\left(\sigma_{1}\right)$ are $G$-equivalent if and only if the involutive automorphisms $\sigma$ and $\sigma_{1}$ are conjugated by an element of $K$. 
3) For any submanifold $M(\sigma)$ there is another canonically defined totally geodesic extrinsically symmetric Kähler submanifold $M\left(\sigma^{\prime}\right)$ associated with the involutive automorphism $\sigma^{\prime}=\varphi_{0} \circ \sigma$ such that one has the orthogonal decomposition $T_{o} W=T_{o} M(\sigma)+T_{o} M\left(\sigma^{\prime}\right)$.

4) The pair of involutive automorphisms $\sigma$ and $\sigma^{\prime}=\varphi_{0} \circ \sigma$ is determined by the restriction of $\sigma$ to $\mathfrak{g}_{0}^{\prime}$. Two automorphisms $\sigma$ and $\sigma_{1}$ define $G$-equivalent pairs $\left(M(\sigma), M\left(\sigma^{\prime}\right)\right)$ and $\left(M\left(\sigma_{1}\right), M\left(\sigma_{1}^{\prime}\right)\right)$ of submanifolds if and only if the automorphism $\left.\sigma\right|_{\mathfrak{g}_{0}^{\prime}}$ is conjugated to $\left.\sigma_{1}\right|_{\mathfrak{g}_{0}^{\prime}}$ or $\left.\sigma_{1}^{\prime}\right|_{\mathfrak{g}_{0}^{\prime}}$ in the group of automorphisms of $\mathfrak{g}_{0}{ }^{\prime}$.

Proof of Theorem. 1) Let $M=L / L_{0}=L o$ be an admissible totally geodesic extrinsically symmetric Kähler submanifold of the Wolf space $W=G / K$ and

$$
\mathfrak{g}=\mathfrak{g}_{-2}+\mathfrak{g}_{-1}+\mathfrak{g}_{0}+\mathfrak{g}_{1}+\mathfrak{g}_{2}=\mathfrak{g}_{\mathrm{ev}}+\mathfrak{g}_{\text {odd }}
$$

the $\operatorname{ad}_{H_{\mu}}$-eigenspace decomposition of the complex Lie algebra $\mathfrak{g}$. We identify the complexified tangent space $T_{o}^{C} W$ with $\mathfrak{p}=\mathfrak{g}_{\text {odd }}=\mathfrak{g}_{-1}+\mathfrak{g}_{1}$.

The symmetry $s_{o}$ of $M$ at point $o$ induces a complex linear involutive transformation $s_{O *}$ of $T_{o}^{C} W=\mathfrak{p}=\mathfrak{g}_{-1}+\mathfrak{g}_{1}$, which by assumption commutes with the complex structure $J_{1}=\left.\operatorname{ad}_{i H_{\mu}}\right|_{\mathfrak{p}}$. This implies that the eigenspace decomposition of $s_{O *}$ has the form

$$
\mathfrak{p}=\left(\mathfrak{g}_{-1}^{+}+\mathfrak{g}_{1}^{+}\right)+\left(\mathfrak{g}_{-1}^{-}+\mathfrak{g}_{1}^{-}\right),
$$

where the +1 -eigenspace $\mathfrak{m}^{C}=\mathfrak{g}_{-1}^{+}+\mathfrak{g}_{1}^{+}$is the complexification of the tangent space $\mathfrak{m}=$ $T_{o} M$ and $\mathfrak{g}_{-1}^{-}+\mathfrak{g}_{1}^{-}$is its orthogonal complement. The graded subspace $\mathfrak{m}^{C}$ generates a graded Lie subalgebra $\ell=\left[\mathfrak{m}^{C}, \mathfrak{m}^{C}\right]+\mathfrak{m}^{C}$ of $\mathfrak{g}$. Since $\left[\mathfrak{m}^{C}, \mathfrak{m}^{C}\right]$ cannot contain the subalgebra $\mathfrak{s p}_{1}^{\mu}(\boldsymbol{C})$, it belongs to $\mathfrak{g}_{-1}+\mathfrak{g}_{0}+\mathfrak{g}_{1}$. In particular, $\left[\mathfrak{g}_{1}^{+}, \mathfrak{g}_{1}^{+}\right]=\left[\mathfrak{g}_{-1}^{+}, \mathfrak{g}_{-1}^{+}\right]=0$. On the other hand, $\ell_{0}=\left[\mathfrak{m}^{C}, \mathfrak{m}^{C}\right] \subset \mathfrak{g}_{0}$ contains $H_{\mu}$, since $M=L / L_{0}$ is a Hermitian symmetric space.

We denote by $\sigma$ the involutive automorphism of the group $G$ and its Lie algebra $\mathfrak{g}^{\tau}$ defined by conjugation with the symmetry $s_{o}$, and extend it to a complex linear automorphism $\sigma$ of $\mathfrak{g}$, which commutes with $\tau$. Since the restriction $\left.\sigma\right|_{\mathfrak{p}}=\left.s_{o}\right|_{\mathfrak{p}}$ commutes with $J_{1}=$ $\left.\operatorname{ad}_{i H_{\mu}}\right|_{\mathfrak{p}}$, we have $\sigma\left(H_{\mu}\right)=H_{\mu}$, that is, $\sigma$ preserves the gradation of $\mathfrak{g}$ defined by $H_{\mu}$. In particular, $\sigma\left(E_{ \pm \mu}\right)=\varepsilon E_{ \pm \mu}$, where $\varepsilon= \pm 1$. Assume that $\varepsilon=+1$, i.e., $\sigma\left(E_{ \pm \mu}\right)=E_{ \pm \mu}$. Then $\left.\left(s_{o}\right)_{*}\right|_{T_{o} W}$ commutes with the quaternionic structure $Q=\operatorname{ad}_{\mathfrak{s p}}^{\mu}(\boldsymbol{C})$, which contradicts the assumption that $M$ is totally complex. Hence $\sigma\left(E_{ \pm \mu}\right)=-E_{ \pm \mu}$. We have proven that the automorphism $\sigma$ defined by the symmetry $s_{O}$ satisfies all conditions of the theorem.

Now we remark that

$$
\left[\mathfrak{g}_{ \pm 1}^{+}, \mathfrak{g}_{ \pm 1}^{+}\right]=\left[\mathfrak{g}_{ \pm 1}^{-}, \mathfrak{g}_{ \pm 1}^{-}\right]=0,
$$

since $\left.\sigma\right|_{\mathfrak{g}_{ \pm 2}}=-$ Id. This means that $\mathfrak{g}_{ \pm 1}=\mathfrak{g}_{ \pm 1}^{+}+\mathfrak{g}_{ \pm 1}^{-}$is a decomposition of the complex symplectic vector space $\mathfrak{g}_{ \pm 1}$, with the symplectic form $\omega$ defined by $[X, Y]=\omega(X, Y) E_{ \pm \mu}$, into direct sum of two Lagrangian subspaces. In particular,

$$
\operatorname{dim} \mathfrak{g}_{1}^{+}=\operatorname{dim} \mathfrak{g}_{1}^{-}=\operatorname{dim} \mathfrak{g}_{-1}^{+}=\operatorname{dim} \mathfrak{g}_{-1}^{-}=\frac{1}{4} \operatorname{dim} W .
$$

Conversely, let $\sigma$ be an involutive automorphism commuting with $\tau$ and acting as - Id on $\mathfrak{g}_{-2}+\mathfrak{g}_{2}$. Then it preserves $H_{\mu}=\left[E_{\mu}, E_{-\mu}\right]$. Hence its eigenspaces decomposition has 
the form

$$
\mathfrak{g}=\mathfrak{g}_{-2}+\mathfrak{g}_{-1}^{+}+\mathfrak{g}_{-1}^{-}+\mathfrak{g}_{0}^{+}+\mathfrak{g}_{0}^{-}+\mathfrak{g}_{-1}^{+}+\mathfrak{g}_{-1}^{-}+\mathfrak{g}_{2} .
$$

Moreover, $\left[\mathfrak{g}_{ \pm 1}^{+}, \mathfrak{g}_{ \pm 1}^{+}\right]=\left[\mathfrak{g}_{ \pm 1}^{-}, \mathfrak{g}_{ \pm 1}^{-}\right]=0$ and the four spaces $\mathfrak{g}_{ \pm 1}^{ \pm}$have the same dimension. One can easily check that the subalgebras

$$
\ell^{-}=\mathfrak{g}_{-1}^{-}+\mathfrak{g}_{1}^{-}, \quad \ell^{+}=\mathfrak{g}_{-1}^{+}+\mathfrak{g}_{1}^{+}
$$

define two totally geodesic extrinsically symmetric Kähler submanifolds $M^{+}=M(\sigma)$ and $M^{-}=M\left(\varphi_{0} \circ \sigma\right)$ of the same dimension $2 n=(1 / 2) \operatorname{dim} W$.

To prove that the correspondence between $\sigma$ and $M(\sigma)$ is a bijection, it is sufficient to show that two involutive automorphisms $\sigma, \sigma^{\prime}$ coincide if they have the same restriction to $\mathfrak{g}_{-1}+\mathfrak{g}_{1}$ or, equivalently, that the fixed point set $\mathfrak{g}^{\sigma}=\mathfrak{g}_{-1}^{\sigma}+\mathfrak{g}_{0}^{\sigma}+\boldsymbol{C} H_{\mu}+\mathfrak{g}_{1}^{\sigma}$ can be reconstructed from $\mathfrak{g}_{-1}^{\sigma}+\mathfrak{g}_{1}^{\sigma}$. Since $\mathfrak{g}_{0}^{\prime}=\left[\mathfrak{g}_{-1}, \mathfrak{g}_{1}\right]$, we have

$$
\mathfrak{g}_{0}{ }^{\prime \sigma}=\left[\mathfrak{g}_{-1}, \mathfrak{g}_{1}\right]^{\sigma}=\left[\mathfrak{g}_{-1}^{\sigma}, \mathfrak{g}_{1}^{\sigma}\right] .
$$

2) If $M(\sigma)$ and $M\left(\sigma_{1}\right)$ are $G$-equivalent, there exists an isometry $k \in K$ such that $k M(\sigma)=M\left(\sigma_{1}\right)$. Then the conjugation by $k$ transforms $\sigma$ into $\sigma^{\prime}$. The converse statement is also clear.

3 ) is obvious. To prove 4), it is sufficient to check that an automorphism $\rho=\sigma^{-1} \circ \sigma^{\prime}$ acting trivially on $\mathfrak{g}_{\mathrm{ev}}=\mathfrak{g}_{-2}+\mathfrak{g}_{0}+\mathfrak{g}_{2}$ is either trivial or equal to $\varphi_{0}$. It follows from the fact that the isometry of $W$ associated to $\rho$ with the fixed point $o$ commutes with the stability subgroup $K$ acting irreducibly on $T_{o} W$.

It is not difficult to describe all automorphisms $\sigma$ of $\mathfrak{g}$ which correspond to totally geodesic extrinsically symmetric Kähler submanifolds $M(\sigma)$ in terms of Kac diagrams, see [GOV]. Here we state only a corollary which we use in the proof of Theorem 1.1.

COROLlary 6.2. Let $W=G / K$ be a Wolf space or its non compact dual. Then, up to an isometry, there exist finitely many totally geodesic extrinsically symmetric Kähler submanifolds of $W$. Any one of them has dimension (1/2) dim W.

PROOF. The claim for Wolf spaces follows from Theorem 6.1. It remains true for non compact dual $W^{\prime}$, since totally geodesic Kähler extrinsically symmetric submanifolds can be characterized as totally geodesic Kähler submanifolds which are normal curvature invariant, and the restriction of the natural one-to-one correspondence between totally geodesic submanifolds of $W$ and $W^{\prime}$ gives a one-to-one correspondence between such submanifolds.

Remark that in a symmetric space $M$ there could be even a continuous number of non equivalent totally geodesic submanifolds of given dimension, for example geodesics in a symmetric space of rank greater than 1 .

\section{Proof of Theorems 1.1 and 1.2.}

ProOF OF THEOREM 1.1. Let $M$ be a curvature invariant maximal Kähler submanifold of a Wolf space or its dual. By Lemma 4.2, $M$ is also normal curvature invariant. Hence 
for any point $x \in M$ there exists an involutive isometry $s_{o}$ such that $\left.s_{o}\right|_{T_{x}} M=-\mathrm{Id}$ and $\left.s_{o}\right|_{T_{x}^{\perp} M}=$ Id, see the proof of Theorem 2.6. This shows that the totally geodesic submanifold $M(x)=\exp \left(T_{x} M\right)$ is an extrinsically symmetric maximal Kähler submanifold. Hence by 6.2, the tangent space $T_{x} M$ belongs to one of the finitely many orbits $\mathcal{V}=G(V) \subset \mathrm{Gr}_{2 n} T(G / K)$. By continuity reason, $M$ is a $\mathcal{V}$-submanifold, where $\mathcal{V}$ is defined by one of the extrinsically symmetric Kähler submanifolds. Since $\mathcal{V}$ is curvature and normal curvature invariant, by applying Naitoh's Theorem 2.8, $M$ is totally geodesic if $\tilde{M} \neq \boldsymbol{H} P^{n}$ or the dual quaternionic hyperbolic space $\boldsymbol{H} H^{n}$ (The last statement for $\tilde{M} \neq \boldsymbol{H} P^{n}$ can also be obtained directly by using Theorem 5.4 and Remark 5.5 of [Na2] for the Grassmannian $G_{2}\left(\boldsymbol{C}^{n+2}\right)$. An elementary proof that $G_{2}\left(\boldsymbol{C}^{n+2}\right)$ does not contain non totally geodesic maximal Kähler submanifolds was given in [ADM]). It is known ([Tsu2]) that any parallel Kähler submanifold of $\boldsymbol{H} \boldsymbol{H}^{n}$ is totally geodesic. This proves Theorem 1.1.

Proof OF TheOREM 1.2. The first claim was proved in Theorem 5.1. Assume that the shape tensor $C \neq 0$. Then by Theorem 5.1, $M^{2 m}$ is a parallel maximal Kähler submanifold of a quaternionic Kähler symmetric space $\tilde{M}^{4 m}$. Theorem 1.1 then implies that $\tilde{M}=\boldsymbol{H} P^{m}$. Now result follows from Tsukada's classification of parallel Kähler submanifolds of $\boldsymbol{H} P^{m}$.

\section{REFERENCES}

[ADM] D. V. Alekseevsky, A. J. Di Scala And S. Marchiafava, Kähler submanifolds of Wolf spaces, Recent advances in geometry and topology, 15-27, Cluj Univ. Press, Cluj-Napoca, 2004.

[AM1] D. V. Alekseevsky And S. MarchiafaVA, Hermitian and Kähler submanifolds of a quaternionic Kähler manifold, Osaka J. Math. 38 (2001), 869-904.

[AM2] D. V. AlEKSEEVsky AND S. MARChiAfAVA, A twistor construction of Kähler submanifolds of a quaternionic Kähler manifold, Ann. Mat. Pura Appl. (4) 184 (2005), 53-74.

[B] J. BERndT, Symmetric submanifolds of symmetric spaces, Proceedings of The Seventh International Workshop on Differential Geometry (KMS Special Session on Geometry) (Taegu, 2002), 1-15, Kyungpook Nat. Univ., Taegu, 2003.

[BENT] J. Berndt, J.-H. EshenburG, H. NAitoh And K. TsukadA, Symmetric submanifolds associated with the irreducible symmetric $R$-spaces, preprint, 2003.

[BRS] F. Burstall, J. RaWnsley and S. Salamon, Stable harmonic 2-spheres in symmetric spaces, Bull. Amer. Math. Soc. (N.S.) 16 (1987), 274-278.

[GOV] V. V. Gorbatsevich, A. L. Onishchik And E. B. Vinberg, Lie groups and Lie algebras, III, Structure of Lie groups and Lie algebras, Encyclopeadia Math. Sci. 41, Springer, Berlin, 1994.

[Na1] H. NAITOH, Isotropic submanifolds with parallel second fundamental forms in symmetric spaces, Osaka J. Math. 17 (1980), 95-110.

[Na2] H. NAITOH, Symmetric submanifolds of compact symmetric spaces, Tsukuba J. Math. 10 (1986), 215242.

[Na3] H. NAITOH, Grassmann geometries on compact symmetric spaces of exceptional type, Japan J. Math. (N.S.) 26 (2000), 157-206.

[Na4] H. NAitoh, Parallel submanifolds of complex space forms I, Nagoya Math. J. 90 (1983), 85-117.

[NT] H. NAKAGAWA AND R. TAKAGI, On locally symmetric Kaehler submanifolds in a complex projective space, J. Math. Soc. Japan 28 (1976), 638-667.

[Str] W. StRÜBING, Symmetric submanifolds of Riemannian manifolds, Math. Ann. 245 (1979), 37-44. 
[Ta] H. TASAKI, Quaternionic submanifolds in quaternionic symmetric spaces, Tohoku Math. J. (2) 38 (1986), 513-538.

[Tak] M. TAKEUCHI, Totally complex submanifolds of quaternionic symmetric spaces, Japan. J. Math. (N.S.) 12 (1986), 161-189.

[Tsu1] K. TsukAdA, Parallel Kaehler submanifolds of Hermitian symmetric spaces, Math. Z. 190 (1985), 129150.

[Tsu2] K. TsuKADA, Parallel submanifolds in a quaternion projective space, Osaka J. Math. 22 (1985), 187-241.

[Wo] J. Wolf, Complex forms of quaternionic symmetric spaces, Complex, contact and symmetric manifolds, 265-277, Progr. Math. 234, Birkhäuser Boston, Boston, Mass., 2005.

THE UNIVERSITY OF HULL

COTTINGHAM ROAD

HU6 7RX, HULL

UNITED KINGDOM

E-mail address: D.V.Alekseevsky@maths.hull.ac.uk

DiPARTIMENTO DI MATEMATICA

UNIVERSITA' DEGLi STUdi Di ROMA “LA SAPIENZA"

P.LE A. MORO 2

00185 ROMA

ITALY

E-mail address: marchiaf@mat.uniroma1.it
Dipartimento di MATEMATICA

POLITECNICO DI TORINO

Corso DuCA DEGLi AbruZzi 24

10129 TORINO

ITALY

E-mail address: antonio.discala@polito.it 